\title{
Assessing the performance of compound smoother in extracting sine signal from heavy noise
}

\begin{abstract}
The $4253 \mathrm{H}$ (Twice) is a type of established non-linear compound smoother that has the capability to extract signal from heavy noise. In this paper, the performance of $4253 \mathrm{H}$ (Twice) in recovering the sine signal with the existence of four different percentage of contaminated error were assessed. The performances are measured by the mean of least square coefficient linear regression of the smoothed sequence on the signal sequence and Estimated Integrated Mean Square Error via simulation process. The $4253 \mathrm{H}$ (Twice) performs best in extracting the signal at low frequency. At the present of $75 \%$ contaminated error, this method manages to recover the signal fairly well. Therefore, $4253 \mathrm{H}$ (Twice) is an effective method in smoothing the heavy noise data.
\end{abstract}

Keyword: Signal; Heavy noise; 4253H (Twice); Smoothing 